\title{
Nueva metodología empírica para la estimación del desgaste de los cortadores de una tuneladora
}

\section{New empirical methodology for TBM cutter wear estimation}

\author{
$\underline{\text { C. Laín }}^{(*)}$, B. Llamas ${ }^{(*)}$, J. Pous $^{(*)}$, R. Laín ${ }^{(*)}$
}

\section{RESUMEN}

El objetivo de este artículo es crear una nueva metodología más sencilla, rápida y barata de las que existen en la actualidad para estimar el desgaste de los cortadores de una tuneladora operando en roca dura, utilizando para ello un desarrollo estadístico que ha permitido establecer una fórmula empírica.

Se han identificado y asignando valores a las diferentes propiedades de las rocas que influyen principalmente en el desgaste de los discos, obteniendo el índice CLH, que estima el desgaste de los cortadores de la cabeza de corte de una tuneladora TBM, pudiendo así prever la duración de los mismos.

Se ha realizado un profundo estudio estadístico de los resultados para demostrar la fiabilidad del método, así como una cuantificación de los posibles errores que se puedan cometer y niveles de confianza.

Palabras clave: TBM; túneles; roca dura; excavación subterránea; cortador; desgaste.

\section{ABSTRACT}

The aim of this article is to create a new methodology that is simpler, faster and cheaper than those that exist today to estimate the wear of the cutters of a TBM operating on hard rock, using a statistical development that has allowed to establish an empirical formula.

They have been identified and assigned values to the different rock properties that mainly influence the wear of the discs, obtaining the CLH index, which estimates the wear of the cutting head cutters of a TBM, thus being able to predict the duration thereof.

A thorough statistical study of the results has been performed to demonstrate the reliability of the method, as well as a quantification of possible errors that can be committed and levels of confidence.

Keywords: Tunnel boring machine, tunnelling, hard rock, Underground excavation; Cutter; wear.

(*) Escuela Técnica Superior de Ingenieros de Minas y Energía. Universidad Politécnica de Madrid. (España)

Persona de contacto/Corresponding author: carlos.lain@upm.es (C. Lain)

ORCID: http://orcid.org/oooo-0oo2-0513-672X (C. Lain); http://orcid.org/oooo-0oo3-4755-5997 (B. Llamas); http:// orcid.org/o000-0002-1777-7073 (J. Pous); http://orcid.org/oooo-0003-4705-5546 (R. Lain)

Cómo citar este artículo/Citation: Laín, C., Llamas, B., Pous, J., Laín, R. (2018). Nueva metodología empírica para la estimación del desgaste de los cortadores de una tuneladora. Informes de la Construcción, 70(549): e242. https://doi.org/10.3989/id.57716.

Copyright: (C) 2018 CSIC. Este es un artículo de acceso abierto distribuido bajo los términos de la licencia de uso y distribución Creative Commons Reconocimiento 4.o Internacional (CC BY 4.0). 


\section{INTRODUCCIÓN}

- Las máquinas tuneladoras (TBM, por su acrónimo en inglés tunnel boring machine) se están empleando cada vez más en la excavación de túneles, principalmente hidráulicos, carreteros y de ferrocarril y con longitudes superiores a los 1,5-2 km (1).

- En la excavación de túneles, galerías y pozos con tbm, las propiedades mecánicas de los testigos de rocas tienen una gran importancia en los resultados que se consiguen, pero las de los macizos rocosos influyen aún más (2).

- La excavación del terreno se realiza empujando contra él unos discos de acero que producen la rotura del terreno por compresión. De esta forma, los fragmentos resultantes tienen forma de laja de algunos centímetros de longitud (3).

Cuando una tbm realiza un túnel en un macizo rocoso formado por rocas abrasivas y resistentes, uno de los principales costes de excavación suele ser el de sustitución de los cortadores de la máquina (Figura 1) (4).

Hoy en día la mayor parte de los macizos rocosos que se encuentran en la naturaleza pueden ser perforados mediante tbm pero los costes de ejecución pueden dispararse de tal forma que resulte económicamente desventajosa la utilización de estas máquinas frente a la técnica de perforación y voladura (5).

Existen distintas causas para el cambio de discos como el gripado, rotura del forro o el desgaste producido por el contacto entre el cortador y la roca. En la obra de los túneles de Guadarrama, se observó que el consumo más importante de cortadores era debido al desgaste frente a otras causas (4).

Los datos utilizados para la realización de este artículo provienen de 204 muestras obtenidas en los siguientes túneles ferroviarios de Alta Velocidad Españoles (AVE):
- Túneles de Guadarrama (Madrid-Segovia), dos túneles paralelos de 9,5 m de diámetro y $28 \mathrm{~km}$ de longitud.

- Túneles de Das Maceiras (Pontevedra), dos túneles paralelos de 9,5 m de diámetro y 8,5 km de longitud.

- Túneles de Pajares (León-Asturias), dos túneles paralelos de 9,5 m de diámetro y 24,6 km de longitud.

Dichas muestras se utilizaron en el cálculo del seguimiento del avance de TBM, mediante distintos ensayos geomecánicos realizados en el Laboratorio de Mecánica de Rocas de la ETS de Ingenieros de Minas y Energía de la Universidad Politécnica de Madrid (ETSIME-UPM).

Gracias a los diferentes ensayos realizados se ha podido establecer una fórmula empírica que permite determinar la duración media del conjunto de discos de la cabeza de corte.

\section{MATERIALES Y MÉTODOS}

El problema de la predicción y la estimación de costes de las TBM ha sido discutido ampliamente en la bibliografía y, muchos métodos de predicción han sido desarrollados y propuestos $(6,7,8,9,10,11)$.

Los métodos generalmente más aceptados son: (i) Método Noruego, (NTNU, desarrollado por la Norwegian Institute of Technology), (ii) el de la Universidad de Colorado (CSM, Colorado School of Mines) y los métodos de los profesores (iii) Z.T. Bieniawski (RME) y (iv) N. Barton (QTBM), adaptados a túneles.

\subsection{Método Noruego (NTNU)}

El método está basado en el estudio de los rendimientos conseguidos por varias TBM en diferentes tipos de rocas (12), y permite determinar el desgaste de cortadores, avances y tiempos de ejecución de túneles mediante TBM.

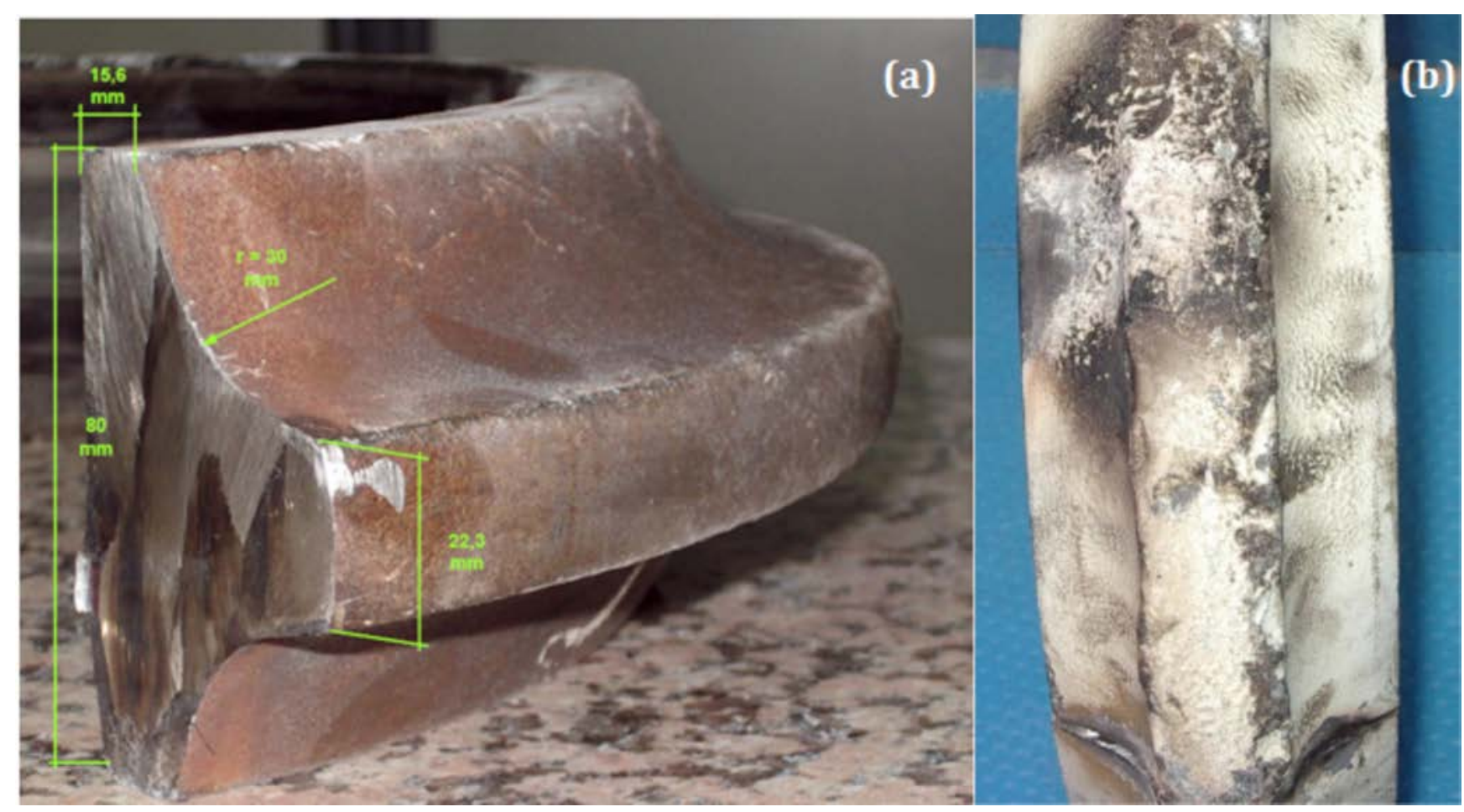

Figura 1. (a) Sección de una herramienta de corte utilizado en una TBM. (b) Desgaste del disco. 
Para ello, considera no sólo las propiedades de las rocas, mediante el DRI (Drilling Rate Index), sino que también las del macizo rocoso.

El índice DRI se desarrolló inicialmente para estimar la velocidad de penetración de martillos de perforación, pero actualmente se considera un buen parámetro para predecir la velocidad de avance de las TBM.

\subsection{Método Colorado}

Este método analiza la influencia de las propiedades de los materiales rocosos en el rendimiento del arranque con TBM (13).

Para ello, calcula o determina mediante ensayos, las fuerzas en los discos y a partir de ellas determinar el empuje total, el par y la potencia requeridas en la cabeza de corte. Estos valores se comparan con los disponibles en las características de la máquina y se obtiene así la máxima penetración que ésta es capaz de conseguir, o de manera inversa, indicar una penetración determinada y ver si los parámetros obtenidos son adecuados para la máquina.

\subsection{Método Barton}

Barton et al. (14), propone una nueva versión de su conocido índice Q para caracterizar los macizos rocoso, cuando se trata de predecir la velocidad neta de avance de una máquina TBM. Este nuevo índice ha sido denominado por su autor QTBM (11).

\subsection{Método Bieniawski}

El profesor Bieniawski presenta el índice de Excavabilidad del Macizo Rocoso, RME (Rock Mass Excavability), para predecir la excavabilidad de mediante TBM (15).

El RME se basa en cinco parámetros relacionados con el comportamiento del macizo rocoso y las características de las tuneladoras para roca.

\subsection{Método CLH}

Este método está basado en la experiencia acumulada en el laboratorio de Mecánica de Rocas de la ETSIME-UPM.

Su máximo objetivo ha sido la simplificación de la determinación del desgaste de los cortadores de una TBM. Para ello, y analizando los resultados de los ensayos se llegó a la conclusión de que los parámetros que más influencia tienen en el desgaste y duración de los discos son (16): la perforabilidad, fragilidad, abrasividad de la roca, el contenido de cuarzo equivalente, la resistencia a compresión simple y el índice de abrasividad cerchar (IAC).

Para la determinación de este índice CLH, se ha considerado:

La duración determinada mediante este método es el factor de utilización, que engloba las paradas de la máquina por cambio de cortadores, acodalamiento, y otros.

El desgaste y por lo tanto la duración de los discos de corte también viene determinada por las características de la máquina y régimen de trabajo, los parámetros de la TBM con los que se ha estimado el desgaste de los cortadores son los siguientes:

- Diámetro cabeza de corte: 9,5 m

- Diámetro de los discos: 432 mm (17")

- Empuje por disco: $200 \mathrm{kN}$

- No de cortadores: 61

- Espaciado de los discos: $75 \mathrm{~mm}$

- Velocidad de giro de la cabeza de corte: $5 \mathrm{rpm}$

\section{ENSAYOS DE LABORATORIO}

En este estudio se han utilizado 204 muestras provenientes de distintos túneles ferroviarios realizados en España mediante TBM, cuyas litologías predominantes son el ortoneis, cataclasitas, pórfidos y distintos tipos de granitos como el biotítico y rosa. Para estas muestras, se realizó la determinación de los parámetros recogidos en el apartado 2.5 y que permitirán definir el índice CLH.

\subsection{Perforabilidad (SJ)}

El ensayo de perforabilidad de Siever (Sievers'J-miniature drill test, SJ) mide la dureza o resistencia a la indentación de la roca (17). Se efectúa con una perforadora de pequeño tamaño, provista de una broca de widia de $8,5 \mathrm{~mm}$ de diámetro y $110^{\circ}$ de ángulo de corte, y se mide la profundidad del agujero que realiza en una muestra de roca después de 200 revoluciones con un empuje de $20 \mathrm{~kg}$ (18). Las medidas tienen que realizarse con una precisión de una décima de milímetro y es necesario efectuar cuatro taladros por muestra.

Este ensayo permite estimar la dureza superficial de la roca y proporciona un índice que se denomina SJ. En la Tabla 1 se muestra la obtención del valor del SJ junto con una fotografía de la muestra después del ensayo.

Tabla 1. Resultados del ensayo de perforabilidad (SJ) para una muestra.

\begin{tabular}{|c|c|c|c|c|}
\hline \multicolumn{4}{|c|}{ Muestra no 4} & \multirow{2}{*}{ 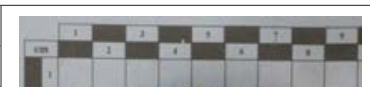 } \\
\hline Testigo & A509V & pk & $8+144$ & \\
\hline Litología & \multicolumn{2}{|c|}{ Granito } & & \\
\hline 1 & \multicolumn{2}{|c|}{2,90} & $\mathrm{~mm}$ & (1) \\
\hline 2 & \multicolumn{2}{|c|}{3,50} & $\mathrm{~mm}$ & $\frac{2}{3}$ \\
\hline 3 & \multicolumn{2}{|c|}{2,90} & $\mathrm{~mm}$ & $\sigma^{2}$ \\
\hline 4 & \multicolumn{2}{|c|}{3,10} & $\mathrm{~mm}$ & \\
\hline Promedio & \multicolumn{2}{|c|}{3,10} & $\mathbf{m m}$ & 2 \\
\hline SJ & \multicolumn{2}{|c|}{31,00} & $1 / 10 \mathrm{~mm}$ & L \\
\hline
\end{tabular}




\subsection{Fragilidad (S2o)}

El ensayo de fragilidad o de impacto da una buena medida de la capacidad de la roca para resistir la trituración por impactos repetitivos, es una propiedad fundamental de la roca y eficaz en la predicción del desgaste de los cortadores de la máquina (9).

Este ensayo proporciona un índice denominado S2O que mide la fragilidad de la roca y expresa de forma indirecta la energía necesaria para romperla (19).

Para ello, se toman 500 g de roca, con una granulometría comprendida entre 16,0 y $11,2 \mathrm{~mm}$, y se colocan en una caja metálica. Sobre este material se hace caer 20 veces desde una altura de $25 \mathrm{~cm}$ una maza de $14 \mathrm{~kg}$ de masa y se determina el porcentaje de material que pasa por el tamiz de 11,2 $\mathrm{mm}$ de malla después del golpeo. Para cada tipo de roca es necesario realizar tres o cuatro pruebas con objeto de obtener una media representativa.

En la Tabla 2 se muestra la obtención del valor del S20 junto con una fotografía de la muestra de roca machacada antes de realizar el ensayo.

\subsection{Abrasión sobre el acero (AVS)}

El ensayo de abrasión sobre el acero (AVS, Abrasion Value Steel) está íntimamente relacionado con el desgaste de los cortadores. La máquina utilizada para este ensayo (Figura 2) mide la abrasión producida por polvo de roca, de granulometría inferior a $1 \mathrm{~mm}$, en un trozo de acero de cortador. El valor del AVS es igual a la pérdida de peso en miligramos de dicha pieza de acero después de 20 revoluciones del plato. Es necesario efectuar dos ensayos por muestra para obtener el AVS (10).

\subsection{Cuarzo equivalente}

El contenido en cuarzo se ha determinado por el método de la lámina delgada, obteniéndose mediante estudio microscópico, así mismo se tiene la composición mineral de la muestra (20).

Se coloca la lámina delgada en la platina de un microscopio con retícula, se cuentan las celdas que contienen cuarzo, se obtiene así el tanto por ciento de este mineral, así como el diámetro de las partículas.

Para el cálculo del cuarzo equivalente se multiplica el tanto por uno de cada mineral de la muestra por el factor de Rosiwall (21), que mide en escala absoluta la dureza de los minerales, se expresa como la resistencia a la abrasión medidas en pruebas de laboratorio tomando como base el corindón con un factor de 1000 , teniendo el cuarzo un factor de 100, se obtiene así el cuarzo equivalente de cada mineral, se suman todos y obtiene la cantidad de cuarzo equivalente (Tabla 3).

Tabla 2. Resultados del ensayo de fragilidad (S2O) para una muestra.

\begin{tabular}{|c|c|c|c|c|}
\hline \multicolumn{4}{|c|}{ Muestra no 3} & \multirow{2}{*}{ i } \\
\hline Testigo & A509H & pk & $8+144$ & \\
\hline Litología & \multicolumn{3}{|c|}{ Granito } & $A$ \\
\hline Ensayo & $1^{a}$ Masa (g) & $2^{a} \operatorname{Masa}(g)^{(1)}$ & Diferencia en \% & 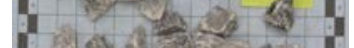 \\
\hline 1 & 501,0 & 253,3 & 50,56 & 3 \\
\hline 2 & 500,0 & 208,9 & 41,78 & 1 \\
\hline \multicolumn{3}{|c|}{ Promedio S2o (\%) } & 46,17 & 2 \\
\hline
\end{tabular}

(1) Masa que pasa por el tamiz de 11,2 mm

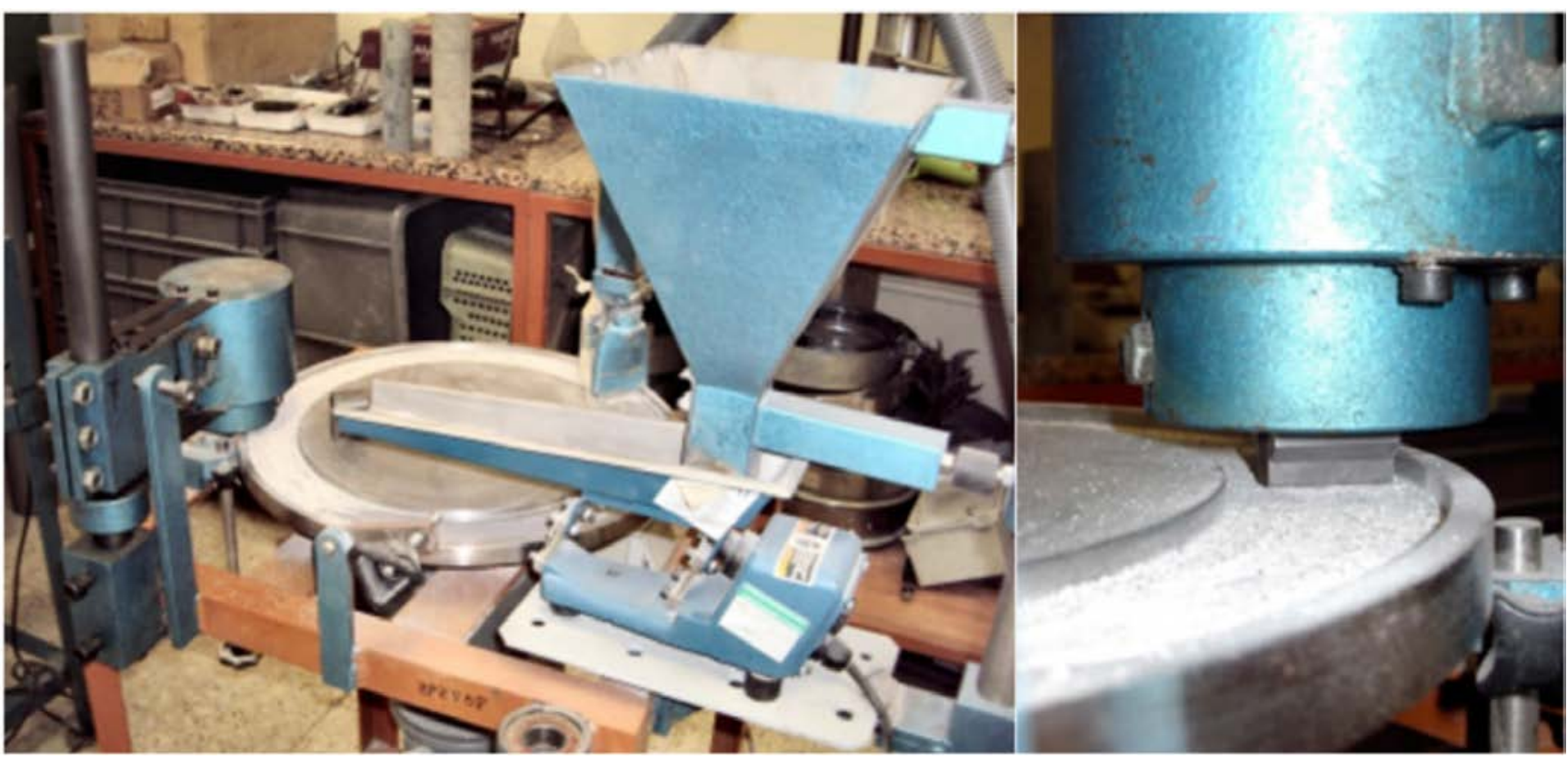

Figura 2. Máquina para ensayos AVS (Laboratorio de Mecánica de Rocas, ETSIME-UPM). 
Tabla 3. Determinación de cálculo del cuarzo equivalente

\begin{tabular}{|l|c|c|c|}
\hline & $\begin{array}{c}\text { Contenido mineral } \\
\text { en tanto por uno }\end{array}$ & $\begin{array}{c}\text { Factor } \\
\text { de Rosiwall }\end{array}$ & Q (\%) \\
\hline Cuarzo & 0,13 & 100 & 13 \\
\hline Feldespato potásico & 0,31 & 35 & 10,85 \\
\hline Plagioclasa & 0,30 & 35 & 10,5 \\
\hline Moscovita & 0,13 & 4 & 0,52 \\
\hline Biotita & 0,12 & 4 & 0,48 \\
\cline { 2 - 4 } & & TOTAL & $\mathbf{3 5}, \mathbf{3 5}$ \\
\cline { 2 - 4 }
\end{tabular}

\subsection{Resistencia a compresión simple}

Este ensayo sirve para determinar la resistencia a compresión uniaxial de una probeta cilíndrica de roca de altura entre el doble y el triple del diámetro $(22,23)$. Normalmente estas probetas se obtienen a partir de testigos de sondeos, aunque también se pueden obtener muestras a partir de bloques de roca mediante una sonda, en el laboratorio.

\section{6. Índice de Abrasividad Cerchar (IAC)}

A partir de este ensayo se obtiene el índice Cerchar para estimar el valor de la abrasividad de las rocas, midiendo el desgaste de un cono de acero causado por el rayado sobre una superficie de roca (24).

Un cono estandarizado bajo un peso constante de $7 \mathrm{~kg}$ raya la superficie de la roca durante un centímetro. Este procedimiento se repite cinco veces en varias direcciones, utilizando cada vez un cono nuevo, se mide a través del microscopio el diámetro de aplanamiento de la punta de cada cono. Cada punto de este índice equivale a un desgaste de $0,1 \mathrm{~mm}$ de la cabeza del cono.

El índice Cerchar (IAC) varía de cero (sin abrasión) a seis (extremadamente abrasivo).

\section{CÁlCULOS PARA LA OBTENCIÓN DEL ÍNDICE CLH}

De todos los ensayos realizados en el Laboratorio de Mecánica de Rocas de la ETSIME-UPM para el seguimiento de distintos túneles excavados mediante TBM en España, se han seleccionado 204, que son los que tienen en común los seis ensayos necesarios para el cálculo del índice CLH, y que han sido descritos en la sección anterior (Ecuación 1).

$$
\begin{aligned}
& \text { Índice } C L H=A+B+C+D+E+F \\
& \text { Ecuación 1. Obtención del índice CLH. }
\end{aligned}
$$

Donde A es un parámetro que se obtiene a través del ensayo de perforabilidad, B el de fragilidad, $\mathrm{C}$ el de abrasión sobre acero, $\mathrm{D}$ el contenido de cuarzo equivalente, $\mathrm{E}$ la resistencia a compresión simple y $\mathrm{F}$ el índice de abrasividad Cerchar.

- A cada uno de los valores obtenidos en los seis ensayos se les ha asignado unos valores matemáticos (Tabla 4). La elección de dichos valores se ha realizado de forma empírica, mediante tanteos sucesivos hasta hacerlos coincidir lo más posible con la duración real del conjunto de discos de la cabeza de corte. De esta forma, el índice CLH varía entre 20 y 100 donde el desgaste será tanto mayor cuanto mayor sea el índice propuesto (Figura 3).

Tabla 4. Asignación de valores de los seis parámetros considerados en la determinación del índice CLH.

\begin{tabular}{|c|c|}
\hline SJ (1/10 mm) & A \\
\hline$<10$ & 5 \\
\hline $11-20$ & 10 \\
\hline $21-30$ & 15 \\
\hline $31-40$ & 20 \\
\hline $41-50$ & 25 \\
\hline $51-60$ & 30 \\
\hline $61-70$ & 35 \\
\hline$>70$ & 40 \\
\hline
\end{tabular}

\begin{tabular}{|c|c|}
\hline S20 (\%) & B \\
\hline $0-20$ & 3 \\
\hline $21-30$ & 4 \\
\hline $31-40$ & 5 \\
\hline $41-50$ & 6 \\
\hline $51-60$ & 7 \\
\hline $61-70$ & 8 \\
\hline $71-80$ & 9 \\
\hline$>80$ & 10 \\
\hline
\end{tabular}

\begin{tabular}{|c|c|}
\hline AVS (mg) & C \\
\hline $0-10$ & 10 \\
\hline $11-20$ & 9 \\
\hline $21-30$ & 8 \\
\hline $31-40$ & 7 \\
\hline $41-50$ & 6 \\
\hline$>50$ & 5 \\
\hline
\end{tabular}

\begin{tabular}{|c|c|}
\hline Qz (\%) & D \\
\hline $0-10$ & 20 \\
\hline $11-20$ & 18 \\
\hline $21-30$ & 16 \\
\hline $31-40$ & 14 \\
\hline $41-50$ & 12 \\
\hline $51-60$ & 10 \\
\hline $61-70$ & 8 \\
\hline $71-80$ & 6 \\
\hline $81-90$ & 4 \\
\hline $91-100$ & 3 \\
\hline
\end{tabular}

\begin{tabular}{|c|c|}
\hline RCS (MPa) & E \\
\hline $0-50$ & 10 \\
\hline $51-100$ & 9 \\
\hline $101-150$ & 8 \\
\hline $151-200$ & 7 \\
\hline $201-250$ & 6 \\
\hline $251-300$ & 5 \\
\hline $301-350$ & 4 \\
\hline$>350$ & 3 \\
\hline
\end{tabular}

\begin{tabular}{|c|c|}
\hline IAC & F \\
\hline$<0,5$ & 10 \\
\hline $0,5-1$ & 9 \\
\hline $1-2$ & 8 \\
\hline $2-3$ & 7 \\
\hline $3-4$ & 6 \\
\hline $4-5$ & 5 \\
\hline $5-6$ & 4 \\
\hline $6-7$ & 3 \\
\hline $7-8$ & 2 \\
\hline$>8$ & 1 \\
\hline
\end{tabular}


Tabla 5. Cuatro ejemplos del cálculo del índice CLH.

\begin{tabular}{|c|c|c|c|c|c|c|c|c|c|c|c|c|}
\hline $\begin{array}{c}\text { SJ } \\
(\mathbf{1} / \mathbf{1 0} \mathbf{~ m m})\end{array}$ & A & S20 (\%) & B & $\begin{array}{c}\text { AVS } \\
\mathbf{( m g )}\end{array}$ & $\mathbf{C}$ & $\begin{array}{c}\text { Contenido } \\
\text { en cuarzo } \\
\text { equivalente (\%) }\end{array}$ & D & $\begin{array}{c}\text { RCS } \\
\text { (MPa) }\end{array}$ & E & IAC & F & $\begin{array}{c}\text { Índice } \\
\text { CLH }\end{array}$ \\
\hline 15,25 & 10 & 33,69 & 5 & 40,5 & 6 & 61,14 & 8 & 186,7 & 7 & 3,83 & 6 & $\mathbf{4 2}$ \\
\hline 9,95 & 5 & 47,6 & 6 & 35,25 & 7 & 44,1 & 12 & 78,94 & 9 & 2,68 & 7 & $\mathbf{4 6}$ \\
\hline 23,79 & 15 & 44,76 & 6 & 41 & 6 & 46,98 & 12 & 122,88 & 8 & 2,55 & 7 & $\mathbf{5 4}$ \\
\hline 33,73 & 20 & 47,01 & 6 & 30,5 & 7 & 45,35 & 12 & 69,85 & 9 & 1,77 & 8 & $\mathbf{6 2}$ \\
\hline
\end{tabular}

\begin{tabular}{|c|c|c|c|c|}
\hline \multicolumn{5}{|c|}{ DESGASTE } \\
\hline $\begin{array}{c}\text { MUY } \\
\text { BANO }\end{array}$ & BANO & MEDIO & ALTO & $\begin{array}{c}\text { MUY } \\
\text { ALTO }\end{array}$ \\
\hline
\end{tabular}

$\begin{array}{lllllllll}20 & 30 & 40 & 50 & 60 & 70 & 80 & 90 & 100 \text { índice CLH }\end{array}$

Figura 3. Desgaste de los discos de la cabeza de corte de una TBM en función del índice CLH.

- En la Tabla 5 se muestra como ejemplo el cálculo de índice CLH para cuatro de las muestras ensayadas.

De acuerdo a la Figura 3, se considera que con las dos primeras muestras se esperaría un desgaste bajo y medio con las dos siguientes.

\section{5- ANÁLISIS ESTADÍSTICO DE LOS RESULTADOS}

Con el fin de validar el índice propuesto y cuantificar los posibles errores, se ha realizado un análisis estadístico. En primer lugar, se ha estudiado si existe relación entre el índice CLH y la duración de los cortadores y que fortaleza tiene dicha relación mediante la covarianza y el coeficiente de correlación.

Seguidamente, se calculará la relación lineal existente entre las dos variables, para ello se realiza un análisis de regresión mediante el método de mínimos cuadrados, obteniendo una ecuación de ajuste y el coeficiente de determinación que indica la precisión del ajuste. Introduciendo el valor del índice CLH en la ecuación se calcula la duración estimada en horas del conjunto de discos de la cabeza de corte, la cual se compara con la real, obteniendo el error cometido, en porcentaje, entre la duración real y la estimada con la metodología propuesta en este artículo para cada una de las muestras.

Por último, se analizan los niveles de confianza y las probabilidades de cometer errores dados mediante la distribución normal, también llamada campana de Gauss-Laplace.

\subsection{Covarianza y coeficiente de correlación}

\subsubsection{Análisis de correlación}

Muchas veces, los diagramas de dispersión no muestran claramente si existe una relación lineal entre dos variables (25), por lo que es necesario medir el grado de asociación de ellas, es decir, calcular un valor numérico que indique el tipo de relación que hay entre ellas.

Los siguientes son los tipos de asociación lineal que puede haber entre dos variables:
- Directa, si la línea recta es creciente (pendiente positiva).

- Inversa, si la línea recta es decreciente (pendiente negativa).

- Inexistente, que es, obviamente, cuando no hay relación entre las variables (pendiente igual a cero).

\subsubsection{Covarianza}

La covarianza es una medida descriptiva que permite determinar el tipo de asociación lineal entre dos variables. La covarianza muestral, Sxy, se obtiene mediante la Ecuación 2, y la media muestral $(\bar{X})$ se muestra en la Ecuación 3 .

$$
S_{x y}=\frac{\sum_{i=1}^{n}\left(x_{i}-\bar{X}\right) \cdot\left(y_{i}-\bar{Y}\right)}{n-1}
$$

Ecuación 2. Cálculo de la covarianza muestral.

$$
\bar{X}=\frac{x_{1}+x_{2}+x_{3}+\cdots+x_{n}}{n}=\frac{\sum_{i=1}^{n} x_{i}}{n}
$$

Ecuación 3. Cálculo de la media muestral.

donde,

$\mathrm{x}_{\mathrm{i}}=$ valor $\mathrm{i}$-ésimo de la variable $\mathrm{x}$

$\bar{X}=$ media muestral de la variable $\mathrm{x}$.

$\mathrm{y}_{\mathrm{i}}=$ valor i-ésimo de la variable y

$\bar{Y}=$ media muestral de la variable y

$\mathrm{n}=$ tamaño de muestra

Se determina la relación entre el índice CLH (variable x) y la duración real de los discos de corte (variable y). Los resultados obtenidos han sido los siguientes:

$$
\begin{aligned}
& \bar{X}=51,92 \\
& \bar{Y}=92,05 \\
& \mathrm{~S}_{\mathrm{xy}}=130,75
\end{aligned}
$$

$\mathrm{Al}$ ser la covarianza mayor que cero, esta indica que existe una relación directa entre ambas variables 


\subsubsection{Coeficiente de correlación}

Aunque la covarianza indica el tipo de relación lineal que hay entre dos variables, no se puede saber la fortaleza de esta relación. Para ello, se propone calcular el coeficiente de correlación (r), utilizado para medir la magnitud de la relación lineal entre dos variables, es decir, indica cuán fuerte o débil es una relación lineal (Ecuación 4).

$$
r=\frac{S_{x y}}{S_{x} \cdot S_{y}}
$$

Ecuación 4. Cálculo del coeficiente de correlación.

Donde:

$\mathrm{S}_{\mathrm{vy}}=$ la covarianza muestral entre las variables $\mathrm{X}$ e $\mathrm{Y}$.

$\mathrm{S}_{\mathrm{x}}=$ desviación estándar muestral de X (Ecuación 5).

$\mathrm{S}_{\mathrm{y}}=$ desviación estándar muestral de $\mathrm{y}$.

$$
S_{x}=\sqrt{\frac{\sum_{i=1}^{n}\left(x_{i}-\bar{X}\right)^{2}}{n-1}}
$$

Ecuación 5. Cálculo de la desviación estándar muestral.

El rango de valores del coeficiente de correlación $\mathrm{r}$ estará comprendido entre $[-1,1]$, siendo tanto más fuerte cuando el coeficiente esté más próximo a los extremos del intervalo. Los resultados obtenidos en este caso son:

$\mathrm{S}_{\mathrm{x}}=8,20$

$\mathrm{S}_{\mathrm{y}}=17,40$

$\mathrm{r}=0,916$

El coeficiente de correlación $r$ de Pearson indica que la relación lineal existente entre las dos variables (índice CLH y duración real) es muy fuerte.

\subsection{Ajuste de la recta y ecuación}

Para determinar la relación lineal entre las dos variables, se va a realizar un análisis de regresión mediante el método de mínimos cuadrados.

El método de mínimos cuadrados se usa para determinar la ecuación de la recta de regresión, es decir, por medio de él se encuentra la única recta que pasa lo más cerca que se puede de todos los puntos (observaciones) ubicados en un diagrama.

Cuando lo que interesa es analizar una relación de causalidad entre dos variables, primero debemos definir cuál de ellas es la variable $\mathrm{Y}$, variable dependiente, y cuál es la variable $\mathrm{X}$, variable independiente.

La variable dependiente Y es la que se busca explicar; en términos estadísticos, es la que se busca estimar o pronosticar. A su vez, la variable independiente $\mathrm{X}$ es la que brinda información para explicar Y que recibe el nombre de variable de predicción.

Para saber si una variable $\mathrm{X}$ es «buena» para explicar la variable Y se calcula el coeficiente de determinación, representado por $\mathrm{r}^{2}$ que es el cuadrado del coeficiente de correlación (determinado en la sección anterior).

Cuanto más cerca esté de 1 , la variable independiente $\mathrm{X}$ será una buena variable para explicar Y. Es decir, es un factor determinante para Y. En contraparte, conforme r2 se acerca a $\mathrm{O}$, indica que $\mathrm{X}$ no es un factor significativo para explicar $\mathrm{Y}$.

Después de obtener el índice CLH para cada una de las muestras, se ha relacionado este valor con la duración real, obteniendo la Figura 4, ajustando una recta de regresión por mínimos cuadrados obteniendo la Ecuación 6, con un coeficiente de regresión $\mathrm{r}^{2}=0,8403$.

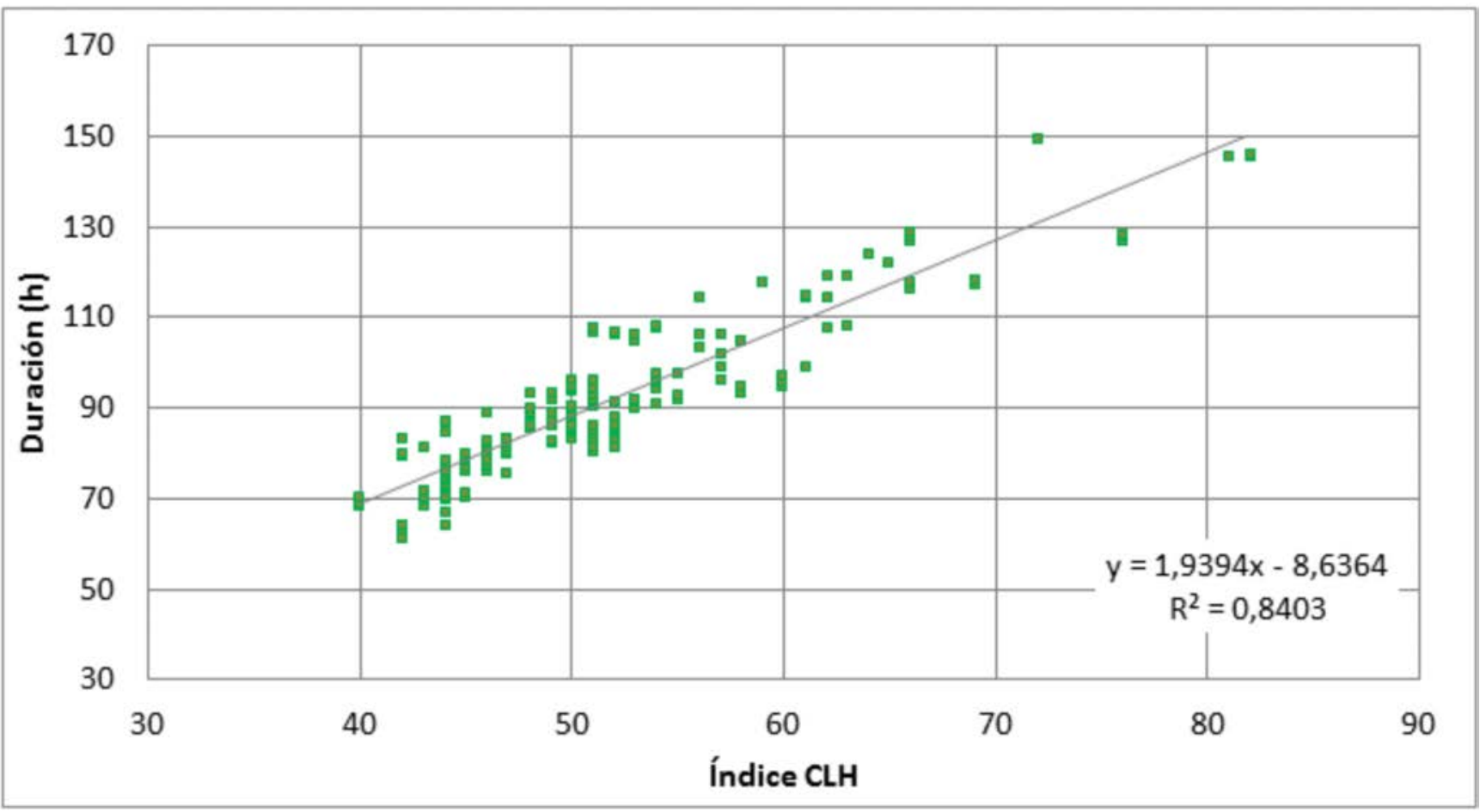

Figura 4. Relación entre la duración real y el índice CLH. 


$$
y=1,9394 \cdot C L H-8,6364
$$

Ecuación 6. Relación entre el índice CLH y la duración real.

Por ejemplo, para una duración real del conjunto de discos de la cabeza de corte de 76,16 h, se ha obtenido un índice CLH de 46 (muestra 2, Tabla 5). Sustituyendo en la Ecuación 6 se obtiene una duración calculada de 80,58 h, una diferencia de $4,42 \mathrm{~h}$, que equivale a un $5,8 \%$.

\section{5•3. Distribución y frecuencia de los resultados}

Obtenidos dichos porcentajes para todas las muestras, estos se pueden agrupar según el porcentaje de error:

- Clase A errores cometidos entre $\pm 2,5 \%$

- Clase B entre $\pm 2,5$ y $\pm 7,5 \%$

- Clase C entre $\pm 7,5$ y $\pm 12,5 \%$

- Clase D entre $\pm 12,5$ y $\pm 17,5 \%$

- Clase E errores mayores de $\pm 17,5 \%$

Si la diferencia es negativa, indica que la duración real es menor que la obtenida y viceversa. En la Figura 5 se muestran las frecuencias y las frecuencias relativas en tanto por ciento de la distribución de los errores cometidos, en 58 muestras se ha cometido un error comprendido entre el $-2,5$ y $+2,5 \%$, Clase A, lo que equivale al $28,43 \%$ de las muestras. Sumando las clases A, $\pm \mathrm{B}$ y $\pm \mathrm{C}$, obtenemos 185 muestras (de un total de 204), que equivale al 90,69\% del total en las que se ha cometido un error igual o inferior al $12,5 \%$.

\subsection{Distribución normal y probabilidades}

Esta distribución, también llamada Campana de GaussLaplace, es frecuentemente utilizada en las aplicaciones estadísticas. Su utilización está justificada por la frecuencia o normalidad con la que ciertos fenómenos tienden a parecerse en su comportamiento a esta distribución.

El modelo de la función de densidad que corresponde a tales distribuciones viene dado por la Ecuación 7.

$$
f(x)=\frac{1}{\sigma \sqrt{2 \pi}} \cdot e^{-\frac{(x-\mu)^{2}}{2 \sigma^{2}}}
$$

donde:

Ecuación 7. Función de densidad.

$\mathrm{f}(\mathrm{x})$ = función de densidad de la variable $\mathrm{x}$, donde $\mathrm{x} \in(-\infty,+\infty)$ $\mathrm{x}$ : valor de la variable a analizar.

$\mu$ : es la media poblacional.

$\sigma$ : es la desviación estándar poblacional.

Los resultados obtenidos han sido, $\mu=-0,59$ y $\sigma=7,3944$.

Son más probables los valores cercanos al central llamado media, $\mu$. Conforme nos separamos de ese valor $\mu$, la probabilidad va decreciendo de igual forma a derecha e izquierda (es simétrica), de forma más o menos rápida dependiendo de un parámetro $\sigma$, que es la desviación típica.

$\mathrm{F}(\mathrm{X})$ es la función de probabilidad acumulada $\mathrm{y}$, se determina mediante la Ecuación 8.

$$
F(x)=\int_{-\infty}^{x} \frac{1}{\sigma \sqrt{2 \pi}} e^{-\frac{(x-\mu)^{2}}{2 \sigma^{2}}} d x
$$

Ecuación 8. Función de probabilidad acumulada.

\subsection{Estandarización}

Si la variable $\mathrm{X}$ es $\mathrm{N}(\mu, \sigma)$ entonces la variable estándar de X es Z, representada en la (ecuación 10), sigue una distribución normal pero de $\mu=$ o y $\sigma=1$, es decir, $N(0,1)$.

$$
Z=\frac{X-\mu}{\sigma}
$$

Ecuación 9. Variable estándar de X.

Su función de densidad es:

$$
f(z)=\frac{1}{\sqrt{2 \pi}} e^{-\frac{z^{2}}{2}}
$$

Ecuación 10. Función de densidad estandarizada.

Su función de probabilidad acumulada es:

$$
F(z)=\frac{1}{\sqrt{2 \pi}} \int_{-\infty}^{z} e^{-\frac{z^{2}}{2}} d z
$$

Ecuación 11. Función de probabilidad acumulada estandarizada.

La distribución de la variable $\mathrm{Z}$ se encuentra en dos tablas: para $Z \geq 0$, y para $Z \leq 0$, (25). Estas indican la probabilidad de encontrar un valor igual o menor que una $\mathrm{Z}$ determinada; por ejemplo, para $Z=1,5$ existe una probabilidad del 93,32\% de que el valor en cuestión sea igual o inferior a 1,5. Con ello, se construye la tabla de probabilidades (Tabla 6).

La variable $\mathrm{X}$ indica la diferencia porcentual entre la duración real y la estimada, la variable $\mathrm{Z}$ se calcula mediante la Ecuación 9, la función de probabilidad acumulada estandarizada $\mathrm{F}(\mathrm{Z})$ se obtiene a partir de la tablas mencionadas anteriormente (25) Por ejemplo, la probabilidad de que el error

\begin{tabular}{|c|c|c|c|}
\hline $\mathbf{X}$ & $\mathbf{Z}$ & $F(Z) \%$ & Probabilidad \% \\
\hline 2,50 & 0,42 & 66,28 & \multirow{2}{*}{26,54} \\
\hline$-2,50$ & $-0,26$ & 39,74 & \\
\hline 5,00 & 0,76 & 77,64 & \multirow{2}{*}{50,21} \\
\hline$-5,00$ & $-0,60$ & 27,43 & \\
\hline 7,50 & 1,09 & 86,21 & \multirow{2}{*}{68,59} \\
\hline$-7,50$ & $-0,93$ & 17,62 & \\
\hline 10,00 & 1,43 & 92,36 & \multirow{2}{*}{82,16} \\
\hline$-10,00$ & $-1,27$ & 10,20 & \\
\hline 12,50 & 1,77 & 96,16 & \multirow{2}{*}{90,79} \\
\hline$-12,50$ & $-1,61$ & 5,37 & \\
\hline 15,00 & 2,11 & 98,26 & \multirow{2}{*}{95,70} \\
\hline$-15,00$ & $-1,95$ & 2,56 & \\
\hline
\end{tabular}
cometido este comprendido entre el $10 \mathrm{y}-10 \%$ es del 82,16\%. $\mathrm{F}(1,43)-\mathrm{F}(-1,27)=92,36 \times 10,20=82,16$, o lo que es lo mismo, la probabilidad de que el error cometido sea superior al $10 \%$ es del 17,84\% (100-82,16).

Tabla 6. Probabilidades dentro de un rango de error. 

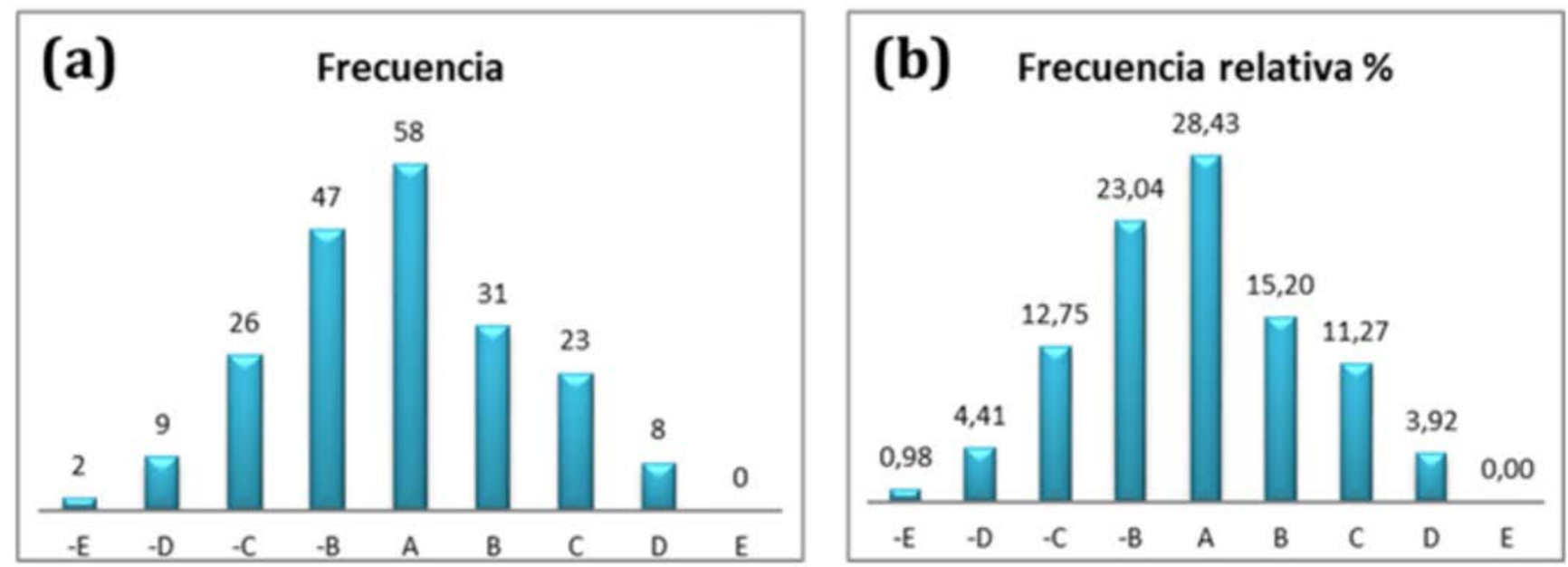

Figura 5. (a) Frecuencias y (b) frecuencias relativas de la distribución de los errores cometidos.

\section{CONCLUSIONES}

Las TBM son utilizadas de una forma amplia para diferentes infraestructuras, especialmente el transporte (tanto automoción como ferrocarril). Estas infraestructuras suelen contar con cuantiosos presupuestos, donde una fuerte incidencia en el mismo deriva de la excavación y de la sustitución de las herramientas de corte.

La sustitución de los cortadores en la TBM depende de diferentes variables, habiéndose demostrado que una de las principales variables es el desgaste de estos.

El cálculo y predicción del avance de TBM es impreciso y depende del tipo de roca atravesada, lo que limita su aplicación en cuanto a su uso como método de estimación del tiempo de desgaste de los cortadores.

El índice CLH no pretende actuar como un sustituto de los distintos métodos para la estimación del desgaste y de la vida útil de los cortadores actualmente utilizados, sino que constituye una herramienta adicional. Existen otras metodologías, como (26) basada únicamente en un parámetro, SJIP, derivado del ensayo de perforabilidad SJ, y (27) que tiene en cuenta el ensayo de abrasividad Cerchar. En esta nueva metodología intervienen seis parámetros relacionados con el desgaste de los cortadores, con lo que se trata de englobar la variabilidad y heterogeneidad inherente de los macizos rocosos.

El índice CLH propuesto en el presente estudio ha demostrado una robustez y suficiente precisión en la estimación del tiempo de desgaste de los cortadores, pudiendo considerase como una nueva y útil herramienta para estimar el desgate de los discos de la cabeza de corte de una TBM (Ecuación 1). Los ensayos necesarios para la determinación del índice son simples y no costosos.

Las diferencias entre las duraciones estimadas y las reales no solo se deben a las intrínsecas al propio método, sino que también a los errores propios asociados en la realización de ensayos de laboratorio y la propia heterogeneidad del macizo rocoso.

El estudio estadístico realizado indica que la relación entre el índice CLH y la duración de los cortadores, mediante el ajuste lineal realizado por mínimos cuadros, es favorable, con un valor de regresión $\mathrm{r}^{2}$ de 0,8403 , cercano a un ajuste perfecto $\left(r^{2}=1,0\right)$.

Los valores siguen una distribución normal pudiendo relacionar los factores de conversión con la probabilidad y viceversa, por lo que se puede obtener la probabilidad de que el factor no supere un cierto valor. Por ejemplo, la probabilidad de que el error cometido este comprendido entre el 10 y $-10 \%$ es del $82,16 \%$.

Por tanto, el índice CLH permite una precisión suficiente como para que esta metodología sea apropiada para una fase preliminar del proyecto, en la que se necesita sólo una idea global y general de los rendimientos de la excavación.

De esta forma, es posible determinar de una forma más precisa el coste derivado de la sustitución de los cortadores, y por tanto mejorar el cálculo de los costes de operación (OPEX) de la infraestructura.

Como próximas líneas de investigación se considera:

Ampliar y completar los ensayos con el fin de analizar y completar el estudio con otro tipo de rocas.

Relacionar litología con los valores obtenidos en los ensayos de perforabilidad (SJ), fragilidad (S2O) y abrasión al acero (AVS).

Determinación del índice CLH para diferentes configuraciones de operación de la maquinaria.

\section{AGRADECIMIENTOS}

A $\mathrm{M}^{\mathrm{a}}$ Elena Madrid Herranz por su ayuda prestada en la realización de este artículo. 


\section{REFERENCIAS}

(1) Salimi, A., Rostami, J., Moormann, C., \& Delisio, A. (2016). Application of non-linear regression analysis and artificial intelligence algorithms for performance prediction of hard rock TBMs. Tunnelling and Underground Space Technology, 58, 236-246.

(2) Ramírez Oyanguren, P., Laín Huerta, R., \& Laín Huerta, C. (2001). Diversas formas de estimar la velocidad de avance de las TBM en rocas. Ingeopres, 99, 16-30.

(3) Sanio, H.P. (1985). Prediction of the performances of disc cutters in anisotropic rock. Int. J. Rock Mech. Min. Sci. \& Geomech. Abstr. 22, 153-161.

(4) Gutiérrez Manjón, J. M. (2006). «Consumo de cortadores de los túneles de Guadarrama». Túnel de Guadarrama. (p. 283-300). Madrid. Ed. Entorno Gráfico.

(5) Lislerud, A. (1988). Hard rock tunnel boring: prognosis and costs. Tunnelling and Underground Space Technology. 3(1), 9-17.

(6) Roxborough, F. F., \& Phillips, H. R. (1975). Rock excavation by disc cutter. International Journal of Rock Mechanics and Mining Sciences \& Geomechanics Abstracts. Pergamon, vol. 12, No. 12, pp. 361-366.

(7) Ozdemir, L., \& Wang, F. D. (1979). Mechanical tunnel boring prediction and machine design. Nasa Sti/Recon Technical Report N, 80, 16239.

(8) Roxborough, F. F. (1985, June). Research in mechanical rock excavation: progress and prospects. In Proc., Rapid Excavation and Tunneling Conference. Society of Mining, Metallurgy, and Exploration, Inc (SME) of the American Institute of Mining, Metallurgical, and Petroleum Engineers (Vol. 1, pp. 225-244).

(9) Bruland, A., 1988. Hard Rock Tunnel Boring, Advance Rate and Cutter Wear, vol. 3 of this Thesis, Norwegian University of Science and Technology, Trondheim, pp. 13-32

(10) Bieniawski, Z. T., Celada, B., \& Galera, J. M. (2007). Predicting TBM Excavatability. Tunnels \& Tunnelling International.

(11) Barton, N., \& Gammelsaeter, B. (2010). Predicting TBM advance using the Q-system and QTBM. Tunnelling Journal (October/November), 32-36.

(12) Bruland, A. (2000). Hard rock tunnel boring (Doctoral dissertation, Fakultet for ingeniørvitenskap og teknologi).

(13) Rostami, J., Ozdemir, L., \& Nilson, B. (1996, May). Comparison between CSM and NTH hard rock TBM performance prediction models. Proceedings of Annual Technical Meeting of the Institute of Shaft Drilling Technology, Las Vegas (pp. 1-10).

(14) Barton, N.R., Lien, R. and Lunde, J. (1974). Engineering classification of rock masses for the design of tunnel support. Rock Mech. 6(4), 189-239.

(15) Bieniawski, Z. T. (1990). Tunnel design by rock mass classifications. Pennsylvania State Univ. University Park. Department of Mineral Engineering.

(16) Laín Huerta, C., Ramirez Oyanguren, P., \& Laín Huerta, R. (2007). Underground Works Under Special Conditions. Chapter 11. The selection of a cutter for a tunnel boring machine and the estimation of its useful life. Proceedings of the ISRM Workshop W1, Madrid, Spain, 6-7 July 2007. Taylor \& Francis 2007. Pages89-96. ISBN: 978-0-415-45028-7. eBook ISBN: 978-0-415-88949-0.

(17) Sievers, H. (1950). Die bestimmung des bohrwiderstandes von gesteinen. Glückauf, 86(7/38), 776-784.

(18) Oggeri, C., Oreste. P. (2012) The wear of tunnel boring machine excavation tools in rock. American Journal of Applied Sciences, 9(10): 1606-1617.

(19) Dahl, F. (2003). DRI, BWI, CLI standards. NTNU, Angleggsdrift, Trondheim.

[20) - (2007). UNE-EN 12407:2007. Métodos de ensayo para piedra natural. Estudio petrográfico. AENOR.

(21) West, G. (1981). A review of rock abrasiveness testing for tunnelling. In ISRM International Symposium. International Society for Rock Mechanics.

(22) Comité AEN/CTN22. UNE 22950-1:1990, propiedades mecánicas de las rocas. Ensayos para la determinación de la resistencia. Parte 1: resistencia a compresión uniaxial. AENOR.

(23) Sub-committee D18.12 (Rock Mechanics). ASTM D7012: Standard Test Methods for Compressive Strength and Elastic Moduli of Intact Rock Core Specimens under Varying States of Stress and Temperatures. ASTM International.

(24) Ghasemi, A. (2010). Study of Cerchar abrasivity index and potential modifications for more consistent measurement of rock abrasion. (Doctoral dissertation, The Pennsylvania State University).

(25) Walpole, R. E., Myers, R. H., \& Myers, S. L. (1999). Probabilidad y estadística para ingenieros. Pearson Educación, pp. 681-682.

(26) Dahl, F., Grøv, E., Breivik, T. (2007). Development of a new direct test method for estimating cutter life, based on the Sievers' J miniature drill test. Tunnelling and Underground Space Technology, 22: 106-116.

(27) Lee, S., Jeong, H.Y., Jeon, S. (2013). Assessment of TBM cutter wear using Cerchar abrasiveness test. Underground-The way to the future, pp. 1209-1216. World tunnel congress (WTC). 